\title{
Measurement of cold challenge responses in primary Raynaud's phenomenon and Raynaud's phenomenon associated with systemic sclerosis
}

\author{
D O'Reilly, L Taylor, K El-Hadidy, M I V Jayson
}

\begin{abstract}
Using computed thermography continuous temperature recordings were made before and after cold challenge of the fingers of control subjects and patients with primary Raynaud's phenomenon and Raynaud's phenomenon associated with systemic sclerosis. Basal skin temperature measurements $\left(T_{\text {pre }}\right)$ were significantly lower in patients with primary Raynaud's phenomenon and Raynaud's phenomenon associated with systemic sclerosis than in the controls. Temperatures immediately after cold challenge $\left(T_{0}\right)$ were significantly lower in patients with primary Raynaud's phenomenon and Raynaud's phenomenon associated with systemic sclerosis than in controls. The lag phase before the start of temperature recovery $\left(T_{\text {lag }}\right)$ was significantly greater in patients with primary Raynaud's phenomenon and Raynaud's phenomenon associated with systemic sclerosis than in control subjects. The maximum recovery index $\left(\mathbf{R}_{\%}\right)$ was significantly less in patients with primary Raynaud's phenomenon and Raynaud's phenomenon associated with systemic sclerosis than in controls. The maximum rate of change of temperature during the rapid phase of rewarming $\left(G_{\max }\right)$ was significantly greater in controls than in patients with primary Raynaud's phenomenon and Raynaud's phenomenon associated with systemic sclerosis. Discriminant analysis showed that the dynamic parameters of rewarming ( $T_{\text {lag}}$, $\mathbf{G}_{\max }$, and $\mathbf{R}_{\%}$ ) showed greater variation between the patients with primary Raynaud's phenomenon and those with Raynaud's phenomenon associated with systemic sclerosis than did $T_{\text {pre }}$ or $T_{0}$. This method of analysis of cold challenge will be used in studies of the effects of treatment of Raynaud's phenomenon.
\end{abstract}

(Ann Rheum Dis 1992; 51: 1193-1196)

In Raynaud's phenomenon there is episodic digital ischaemia with phasic colour changes on exposure to cold or emotional stress. Although the clinical appearances are well recognised it has proved difficult to quantify the changes in the microcirculation. Using modern techniques of thermography and computed image analysis it is possible to make continuous real time recordings of the rewarming curves of subjects after a cold challenge. We have used these techniques to measure the differing microcirculatory response to cold challenge in control subjects and patients with primary Raynaud's phenomenon and Raynaud's phenomenon associated with systemic sclerosis.

\section{Subjects and methods}

Raynaud's phenomenon is defined as at least two years of abnormal responses to exposure to cold with skin colour changes of pallor followed by cyanosis or erythrocyanosis, or both. The patients with primary Raynaud's phenomenon had no history, physical signs, nor serological features suggestive of connective tissue disease. The patients with Raynaud's phenomenon associated with systemic sclerosis fulfilled the diagnostic criteria of the American Rheumatism Association for systemic sclerosis. ${ }^{1}$

There were 21 control subjects (14 women, seven men; mean age $39 \cdot 2$ years, range 29-71), 16 patients with primary Raynaud's phenomenon ( 12 women, four men; mean age $44 \cdot 18$ years, range 24-72) and 20 patients with Raynaud's phenomenon associated with systemic sclerosis (19 women, one man; mean age 49.63 years, range $24-67$ ) (table 1 ).

The subjects were asked not to smoke or to take any drugs from midnight before the study. They were rested for 20 minutes in a controlled environment at a temperature of $23 \pm 0.5^{\circ} \mathrm{C}$ and humidity $45 \pm 5 \%$. The right hand was then placed in a frame such that the position of the fingers was fixed and using an Inframetrics $600 \mathrm{~m}$ thermography camera a baseline image of the dorsal surface was recorded onto a video tape. A disposable latex glove was then put on and the hand immersed in water at $15^{\circ} \mathrm{C}$ for one minute. After removal of the glove the hand was returned to the same position. Continuous thermographic video tape recordings were made for 15 minutes.

Using the commercially available Thermotechnics TTX2 thermography program on a standard IBM microcomputer three points were selected over the distal interphalangeal joints of the index, middle, and ring fingers during replay of the video taped recording. The temperature at each point was plotted at 15 second intervals as rewarming curves.

Table 1 Characteristics of subjects studied

\begin{tabular}{lll}
\hline Subjects $^{*}$ & $\begin{array}{l}\text { Male/female } \\
\text { ratio }\end{array}$ & $\begin{array}{l}\text { Mean (range) } \\
\text { age (vears) }\end{array}$ \\
\hline Controls $(\mathrm{n}=21)$ & $7 / 14$ & $39 \cdot 2(24-71)$ \\
PRP $(\mathrm{n}=16)$ & $4 / 12$ & $44 \cdot 2(24-72)$ \\
SSRP $(\mathrm{n}=20)$ & $1 / 19$ & $49 \cdot 6(24-67)$ \\
\hline
\end{tabular}

PRP=primary Raynaud's phenomenon; SSRP=Raynaud's phenomenon associated with systemic sclerosis. 


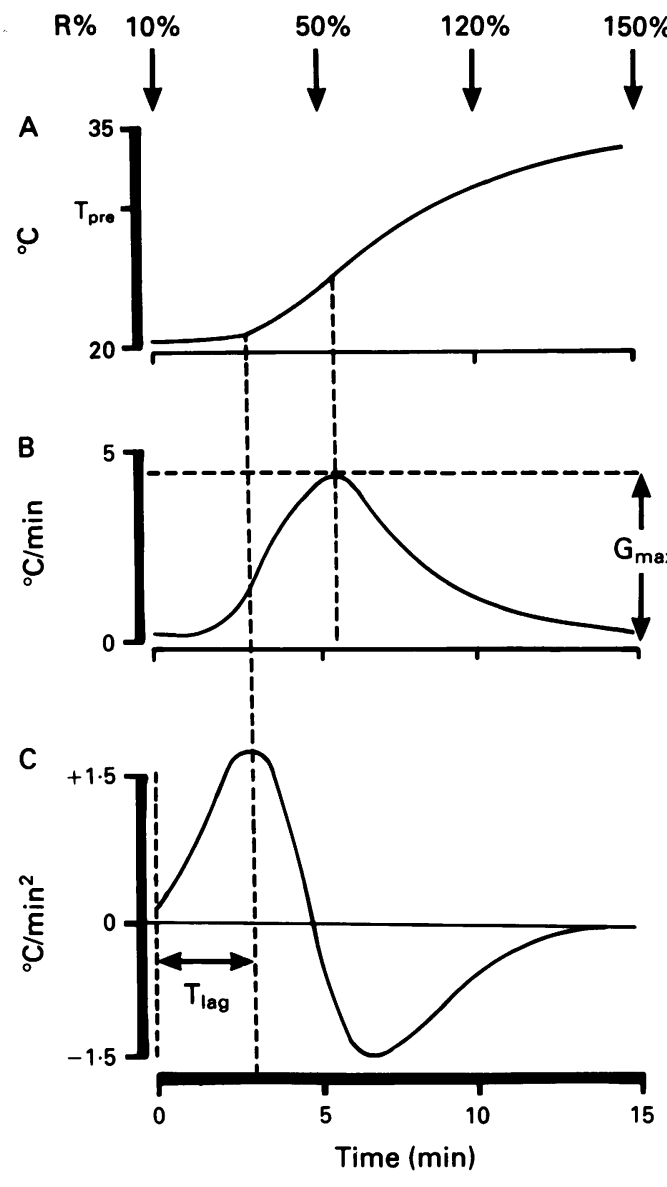

(A) Typical rewarming curve of a control subject; arrows indicate recovery index at $1,5,10$, and 15 minutes. (B) First differentiation of rewarming curve to estimate $G_{\text {max }}$, the maximum temperature gradient. (C) Second differentiation maximum temperature gradient. (C) Second differentiation
of rewarming curve to estimate $T_{\text {lu, }}$ the lag phase before the rapid phase of rewarming. $R \%=$ recovery index.

The temperature recovery curves (figure) following a cold challenge typically showed a lag phase when there was little alteration in temperature followed by a phase of rapid increase in temperature. In normal subjects the temperature usually exceeded the baseline temperature after the rapid rewarming phase but in patients with primary Raynaud's phenomenon and Raynaud's phenomenon associated with systemic sclerosis temperatures usually did not reach baseline values by 15 minutes after cold challenge.

For each subject the mean temperature decrease on cooling was measured. At 1, 5, 10 , and 15 minutes after cold challenge an index of percentage recovery was calculated (index $=($ temperature increase/initial temperature decrease) $\times 100 \%$ ). The maximum value of this recovery index $\left(\mathbf{R}_{\%}\right)$ was recorded in each subject (figure). Using a locally written program the first differential of the temperature-time curve was plotted. This allowed measurement of the change in temperature with time $\left({ }^{\circ} \mathrm{C} /\right.$ minute) and from this the maximum temperature recovery rate $\left(G_{\max }\right)$ could be measured. This curve was redifferentiated to give the rate of change of temperature gradient with time. The peak of this second differentiation curve indicated the point at which there was the maximum increase in the rate of change of temperature $\left({ }^{\circ} \mathrm{C} / \mathrm{minute} / \mathrm{minute}\right)$ and the time at which this occurred $\left(T_{\text {lag }}\right)$ represented the lag phase from cold challenge until rewarming began.

Using the SPSS statistical program the temperature measurements and $\mathbf{R}_{\%}$ were analysed using Student's $t$ test; the $G_{\max }$ and $T_{\text {lag }}$ measurements were not normally distributed and so were transformed to $\log _{10}$ values before $t$ test analysis. Discriminant analysis (Wilks' method minimising lamda) was performed on all these variables to estimate which parameters best discriminated between the subject groups.

\section{Results}

The mean basal temperature $\left(\mathrm{T}_{\text {pre }}\right)$ was lower in patients with primary Raynaud's phenomenon and Raynaud's phenomenon associated with systemic sclerosis than in controls (table 2). These differences were statistically significant. The mean basal temperature in patients with Raynaud's phenomenon associated with systemic sclerosis was not lower than that in patients with primary Raynaud's phenomenon. The absolute temperature immediately after cold challenge $\left(T_{0}\right)$ was significantly higher in controls than in patients with primary Raynaud's phenomenon and Raynaud's phenomenon associated with systemic sclerosis; however, there was no difference in $T_{0}$ between the patients with primary Raynaud's phenomenon and Raynaud's phenomenon associated with systemic sclerosis.

In controls there was a short lag phase and then a rapid recovery to above basal levels, reflected by the significant differences in $G_{\max }$, $T_{\text {lag }}$, and $R_{\%}$ compared with the other groups. In patients with primary Raynaud's phenomenon there was a longer lag phase with slower and incomplete temperature recovery. In patients with Raynaud's phenomenon associated with systemic sclerosis there was a prolonged period without rewarming with no definite phase. of rewarming, and often no significant recovery by 15 minutes. In these subjects the lag time was

Table 2 Results of cold challenge in subjects studied

\begin{tabular}{|c|c|c|c|c|c|c|}
\hline & \multirow{2}{*}{$\begin{array}{l}\text { Control subjects } \\
(n=2 I)\end{array}$} & \multirow{2}{*}{$\begin{array}{l}\text { Patients with } \\
\text { PRI: } \\
(n=16)\end{array}$} & \multirow{2}{*}{$\begin{array}{l}\text { Patients } \\
\text { with SSRP } \\
(n=20)\end{array}$} & \multicolumn{3}{|l|}{ p Values } \\
\hline & & & & $\begin{array}{l}\text { Controls } \\
v P R P\end{array}$ & $\begin{array}{l}\text { PRP v } \\
\text { SSRP }\end{array}$ & $\begin{array}{l}\text { Controls } \\
v S S R I\end{array}$ \\
\hline \multirow[t]{2}{*}{$\begin{array}{l}\text { Age (years) } \\
T_{\text {rre }}\left({ }^{\circ} \mathrm{C}\right) \\
T_{=0}\left({ }^{\circ} \mathrm{C}\right) \\
\log _{10} \mathrm{G}_{\text {max }} \\
\log _{10,} \mathrm{~T}_{\text {lig }} \\
\text { Maximum observed recovery } \\
\text { index }(\%)\end{array}$} & $\begin{array}{l}39 \cdot 2 \\
30 \cdot 04 \\
22 \cdot 6 \\
0 \cdot 2351 \\
0 \cdot 1934\end{array}$ & $\begin{array}{l}44 \cdot 2 \\
25 \cdot 9 \\
20 \cdot 02 \\
-0 \cdot 16 \\
0.654\end{array}$ & $\begin{array}{l}49 \cdot 6 \\
25 \cdot 69 \\
20 \cdot 16 \\
-0 \cdot 353 \\
0 \cdot 982\end{array}$ & $\begin{array}{l}0.006 \\
0.004 \\
0.006 \\
0.005\end{array}$ & $\begin{array}{l}0.86 \dagger \\
0.84 \dagger \\
0.12 \dagger \\
0.02 \dagger\end{array}$ & $\begin{array}{l}0.001 \\
0.002 \\
<0.001 \\
<0.001\end{array}$ \\
\hline & $131 \cdot 2$ & 66.6 & $40 \cdot 35$ & 0.001 & $0.049+$ & $<0.001$ \\
\hline
\end{tabular}

${ }^{*} \mathbf{P R P}=$ primary Raynaud's phenomenon; SSRP=Raynaud's phenomenon associated with systemic sclerosis. †Not significant. 
recorded as greater than 15 minutes and calculated as 15 minutes in the statistical tests. The lag times were longer in patients with Raynaud's phenomenon associated with systemic sclerosis than in those with primary Raynaud's phenomenon, though this did not achieve statistical significance $(p=0.02)$. The maximum observed temperature recovery rate $\mathbf{R}_{\%}$ was significantly lower in patients with primary Raynaud's phenomenon than in controls. $\mathbf{R}_{\%}$ was lower in patients with Raynaud's phenomenon associated with systemic sclerosis than in those with primary Raynaud's phenomenon but again this did not achieve statistical significance $(p=0.049) . G_{\max }$ did not significantly differ between patients with primary Raynaud's phenomenon and Raynaud's phenomenon associated with systemic sclerosis $(p=0 \cdot 841)$. Discriminant analysis of these five measurements in the primary Raynaud's phenomenon and Raynaud's phenomenon associated with systemic sclerosis groups showed that the dynamic parameters $T_{\text {lag }}, G_{\max }$, and $R_{\%}$ were more effective discriminants between patients with primary Raynaud's phenomenon and Raynaud's phenomenon associated with systemic sclerosis than the temperature measurements $T_{\text {pre }}$ and $T_{0}$. Using the discriminant analysis statistical model to predict actual group membership from the original data recorded from the patients with primary Raynaud's phenomenon and Raynaud's phenomenon associated with systemic sclerosis gave correct classification in $75 \%$ of cases with a sensitivity of $80 \%$ and a specificity of $68 \%$.

\section{Discussion}

Continuous recording of the temperature changes in the skin using modern thermographic techniques with computed analysis provides a rapid and relatively simple method of quantifying Raynaud's phenomenon. Earlier techniques include plethysmography and the application of thermistors. These methods will produce local disturbance of the circulation and influence results. Point measurements can be made using a bolometer but this must be applied separately to each site, again disturbing the local microcirculation and introducing artifacts. The thermographic pictures are taken from a distance and provide high resolution with accurate recording of surface temperature. There is no local interference with the microcirculation and so the method is ideal for studying the temperature changes after cold challenge. ${ }^{2}$ The thermographic images are recorded onto video tape for later analysis. Sophisticated computer analysis of the images allows the continuous recording of surface temperature at selected points rather than calculation of the mean temperature of the whole image. The new methods of image analysis allow rapid measurement of these changes in a way suitable for clinical study.

Cold challenge has been reviewed elsewhere. ${ }^{3}$ There is no agreed protocol for cold challenge; however, the experimental conditions used in our study are similar to those widely used elsewhere. ${ }^{3}$ The environmental conditions for this study were chosen to be neither vaso- constrictive nor vasodilatory. The cold challenge (water bath at $15^{\circ} \mathrm{C}$ for one minute) used in this study was relatively mild but seems adequate for quantitative measurements of temperature recovery patterns. The severity of cold challenge in other studies varies from immersion in ice water', ${ }^{4}$ to challenge at $20^{\circ} \mathrm{C} .{ }^{5}$ The more severe challenges are unpleasant for patients and potentially dangerous for those with severe Raynaud's phenomenon.

It is well known that subjects with Raynaud's phenomenon have abnormal cold challenge recovery curves, with lower basal temperatures and slower recovery. ${ }^{3}$ Until now, however, no attempt has been made to use cold challenge to discriminate between patients with primary Raynaud's phenomenon and those with Raynaud's phenomenon associated with systemic sclerosis. As Raynaud's phenomenon is often the first presenting symptom of systemic sclerosis the ability to discriminate between patients with primary Raynaud's phenomenon and Raynaud's phenomenon associated with systemic sclerosis would be a significant clinical advantage. The aim of this study was to take the data from standard cold challenge and to identify objective and quantifiable parameters of the response curves which relate in some way to the pathophysiological processes and can be used to discriminate patients with primary Raynaud's phenomenon from those with Raynaud's phenomenon associated with systemic sclerosis. In normal subjects rewarming begins in the finger pulps with the opening of arteriovenous shunts; the rewarming effect spreads proximally to the palm. In subjects with Raynaud's phenomenon, however, rewarming often starts at the base of the fingers and spreads up to the tips of the fingers; hyperaemia of the finger tips often does not occur. In following the cold challenge response curves a typical pattern is seen (figure). After cold challenge there is often a lag period when the skin surface warms slowly, if at all. There is then a phase of more rapid temperature change before the final phase when skin rewarming has stopped. Most of the controls are hyperaemic in this final phase with a recovery index greater than $100 \%$. In the Raynaud's phenomenon associated with systemic sclerosis group most subjects had relatively flat curves; however, some did show temperature recovery. We used a simple microcomputer program to quantify objectively those characteristics of the recovery curves. The interval before rewarming begins, $T_{\text {lag }}$, was significantly longer in patients with primary Raynaud's phenomenon than in controls. In many subjects with Raynaud's phenomenon associated with systemic sclerosis the rewarming was so slow that the lag time exceeded the length of the 15 minute study period. The rapid rewarming phase seen in most of the controls was also present in most, but not all, of the patients with primary Raynaud's phenomenon. This phase must correspond to a period of increased blood flow through the finger or a change in the distribution of that flow, either through the cutaneous microcirculation or through the deeper vessels with rewarming of the skin by conduction. $G_{\max }$ was derived as a means of quantifying this 
aspect of the rewarming curve and so must relate indirectly to the rate and distribution of blood flow through the finger. $G_{\max }$ was significantly greater in controls than in patients with primary Raynaud's phenomenon. $G_{\max }$ was greater in patients with primary Raynaud's phenomenon than in those with Raynaud's phenomenon associated with systemic sclerosis, but this did not achieve significance. The temperatures immediately before $\left(T_{\text {pre }}\right)$ and after $\left(T_{0}\right)$ cold challenge were similar in patients with Raynaud's phenomenon associated with systemic sclerosis and primary Raynaud's phenomenon. Discriminant analysis of all the variables in the patients with primary Raynaud's phenomenon and those with Raynaud's phenomenon associated with systemic sclerosis showed that the dynamic parameters were more powerful discriminants than $T_{\text {pre }}$ or $T_{0}$. The recovery index $\left(\mathbf{R}_{\%}\right)$ was significantly greater in controls than in patients with primary Raynaud's phenomenon but did not differ in primary Raynaud's phenomenon and Raynaud's phenomenon associated with systemic sclerosis. This measurement is less useful in those subjects with Raynaud's phenomenon associated with systemic sclerosis where $T_{\text {pre }}$ is only 1 or $2{ }^{\circ} \mathrm{C}$ above the cold challenge water temperature. Rewarming of the skin surface by convection alone could then produce an $\mathbf{R}_{\%}$ value close to $100 \%$.

Rather than compare serial measurements of absolute temperature changes in subjects at sequential time points we have compared individual maximum dynamic responses. The statistical problems of analysing serial measurements ${ }^{6}$ are avoided and it is also possible to compare subjects with responses over different temperature ranges.

We found significant reductions in basal skin temperature compared with controls not only in patients with Raynaud's phenomenon associated with systemic sclerosis but also in those with primary Raynaud's phenomenon. Simple measurements of basal temperatures, however, are the same in patients with primary Raynaud's phenomenon and those with Raynaud's phenomenon associated with systemic sclerosis. We have also shown a significant reduction in the rate of increase in skin temperature in the rapid rewarming phase after cold challenge. This may reflect some permanent vascular compromise in primary Raynaud's phenomenon and thus implies that Raynaud's phenomenon is not completely reversible. Alternatively, this could be due to a greater degree of vasoconstriction in the primary Raynaud's phenomenon group at $23^{\circ} \mathrm{C}$ which persists during recovery from cold challenge.

A number of mechanisms have been suggested for the development of Raynaud's phenomenon These include prolonged vasoconstriction, permanent narrowing of the digital arteries, and hyperviscosity of the blood. ${ }^{9}$ The lower basal temperatures in patients with primary
Raynaud's phenomenon are compatible with the hypothesis that there is a permanent impairment of the microcirculation and that Raynaud's phenomenon is not entirely due to reversible vasoconstriction. The prolonged lag phase in patients with primary Raynaud's phenomenon and particularly in those with Raynaud's phenomenon associated with systemic sclerosis may be associated with arterial narrowing or with a disproportionate increase in blood viscosity on cooling. It is known that there is hyperviscosity of the blood in Raynaud's phenomenon associated with systemic sclerosis and primary Raynaud's phenomenon and this increases on cooling. ${ }^{9}$

The technique of quantitative thermography and analysis of rewarming curves appears promising for measurement of the disturbance of the microcirculation in Raynaud's phenomenon and in differentiating between Raynaud's phenomenon associated with systemic sclerosis and primary Raynaud's phenomenon. The method is simple and all measurements and analyses take less than an hour. The technique of computed thermography measures the patient's actual observation that after cold exposure their fingers warm up slowly after a long delay.

The dynamic parameters reflect the characteristics of the individual rewarming curves and will be investigated as indicators of severity of disease and efficacy of treatment in future studies. The relation between $\mathrm{G}_{\max }$ and red cell flux through the microcirculation will be investigated by simultaneous thermography and laser Doppler studies.

We acknowledge the help and advice of Dr D Taylor who wrote the program for differentiation of the rewarming curves and Ms S Hollis for her statistical advice. We are grateful to the Raynaud's and Scleroderma Association for financial support to purchase the computed image analysis system.

1 Subcommittee for scleroderma committee of the American Rheumatism Association diagnostic and therapeutic criteria committee. Preliminary criteria for the classification of systemic sclerosis (scleroderma). Arthritis Rheum 1981; 23 . $581-90$.

2 Darton $\mathrm{K}$, Black $\mathrm{C} M$. The use of infra-red thermography in a rheumatology unit. Br 7 Rheumatol 1990; 29: 291-2.

3 Cooke E D, Nicholaides A N, Porter J M, eds. Raynaud' syndrome. London: Med-Orion, 1991: 99-110.

4 White C J, Phillips W A, Abrahams L A, Watson J T, Singleton Jr P T. Objective benefit of nifedipine in the treatment of Raynaud's phenomenon-a double-blind controlled study. Am 7 Med 1986; 80: 623-5.

5 Rademaker M, Cooke E D, Almond N E, et al. Comparison of intravenous infusions of iloprost and oral nifedipine in treatment of Raynaud's phenomenon in patients with systemic sclerosis: a double blind randomised study. $B M \mathcal{F}$ 1989; 298: $561-4$.

6 Matthews J N S, Altman D G, Cambell M J, Royston P. Analysis of serial measurements in medical research. $B M \mathcal{J}$ 1990; 300: 230-5

7 Lafferty K, Roberts V C, De Trafford J C, Cotton L T. On the nature of Raynaud's phenomenon: the role of histamine. Lancet 1983; ii: 313-5.

8 Rodnan G P, Myerowitz R L, Justh G O. Morphological changes in the digital arteries of patients with progressive systemic sclerosis (scleroderma) and Raynaud's phenomenon. Medicine (Baltimore) 1980; 59: 393-408.

9 Blunt R J, George A J, Hurlow A, Strachan C J L, Stuart J Hyperviscosity and thrombotic changes in idiopathic and secondary Raynaud's syndrome. Br $\mathcal{F}$ Haematol $1980 ; 45$; 651-7. 